\title{
Downton-Mania. Die Fernsehserie „Downton Abbey“ und ihre Auswirkung auf die Public History und den Filmtourismus
}

\author{
Verena Hechenblaikner \\ Kerngebiet: Neuzeit \\ eingereicht bei: assoz. Prof. Dr. Elena Taddei \\ eingereicht im: WiSe 2019/20 \\ Rubrik: Seminar-Arbeit (Vertiefung)
}

\begin{abstract}
Downton Mania. The Television Series "Downton Abbey" and its Impact on Public History and Film Tourism
\end{abstract}

The following seminar-paper examines the potential of historical television series for historical science. Using the television series "Downton Abbey" as an example, it shows series' effects on public history and film tourism. The main argument is that historical series increase public interest in history and thus make it possible to communicate historical topics to a broader audience.

\section{Einleitung}

Histotainment hat sich in den letzten Jahrzehnten zu einem wichtigen Schlagwort in der Darstellung und Vermittlung von Geschichte entwickelt.' So erlebt das öffentliche Interesse an der Vergangenheit seit den 1980er-Jahren einen Boom und Geschichte entwickelt sich zunehmend zu einem "Gegenstand populärkultureller Repräsentation, Produktion und Konsumption"2. Es geht darum, Vergangenes für ein Laien-Publikum in populärer Weise aufzubereiten, wobei neben der Informationsvermittlung hauptsächlich Spannung und Unterhaltung im Vordergrund

\footnotetext{
Thomas Fischer/Thomas Schuhbauer, Geschichte in Film und Fernsehen. Theorie-Praxis-Berufsfelder (Public History-Geschichte in der Praxis), Tübingen 2016, S. 99.

2 Barbara Korte/Sylvia Paletschek, Geschichte in populären Medien und Genres. Vom historischen Roman zum Computerspiel, in: Barbara Korte/Sylvia Paletschek (Hrsg.), History Goes Pop. Zur Repräsentation von Geschichte in populären Medien und Genres (Historische Lebenswelten in populären Wissenskulturen/History in Popular Cultures 1), Bielefeld 2009, S. 9-60, hier S. 9.
} 
stehen. ${ }^{3}$ Dementsprechend sind historische Darstellungen in den unterschiedlichsten Medien allgegenwärtig. Ein gesamtgesellschaftlicher Trend zeichnet sich vor allem in der filmischen Darbietung der Vergangenheit ab. ${ }^{4}$ So gehören "das Kino und das Fernsehen zu den wirkungsmächtigen Medien, in denen Geschichtsbilder [...] heute einem Breitenpublikum vermittelt werden“5. Daraus resultiert vielfach der Wunsch, die Drehorte zu besuchen ${ }^{6}$, was in weiterer Folge die Public History und den Filmtourismus beeinflusst: " "The growing pull of heritage has made the past the world's most popular tourist destination."

Die vorliegende Seminararbeit setzt an diesem Punkt an und beschäftigt sich mit den Auswirkungen der britischen Fernsehserie „Downton Abbey“. Dabei werden zwei Fragestellungen verfolgt: Zum einen soll allgemein untersucht werden, ob Szenische Historienserien ${ }^{9}$, die primär auf Unterhaltung ausgelegt sind, für die Geschichtswissenschaft von Nutzen sein können. Zum anderen geht die Arbeit der Frage nach, welche Auswirkungen "Downton Abbey" auf die Public History und den Filmtourismus hat. Das Ziel besteht darin, diese Folgen an ausgewählten Beispielen exemplarisch zu veranschaulichen und dadurch auf den geschichtswissenschaftlichen Nutzen von Szenischen Historienserien zu schließen. Die leitende These der Arbeit ist folgende: Die britische Fernsehserie „Downton Abbey" zeigt, dass Szenische Historienserien ein mögliches Medium sind, um in der Öffentlichkeit Interesse an Geschichte zu wecken. Dieses Interesse können die Public History und der Filmtourismus nutzen, wodurch es gelingen kann, einem breiten Publikum historische Themen zu vermitteln und gegebenenfalls auch historische Gebäude zu erhalten.

Zur Bearbeitung des Themas wird sowohl auf Fachliteratur als auch auf Internetquellen zurückgegriffen. So dienen die beiden Publikationen „Einführung in die Public History“10 und "Filmtourismus"11 als Grundlagenwerke, da sie einen Einblick in das jeweilige Forschungsfeld bieten. Die Arbeiten von Andrew Higson sind, mit ihrer detaillierten Beschreibung der britischen Filmgeschichte, ebenso zentral. ${ }^{12}$ Als weitere wichtige Veröffentlichungen, welche sich explizit mit der Serie „Downton Abbey“ auseinandersetzen, können die Monographie "Downton Abbey on Location“13 sowie der wissenschaftliche Aufsatz ,The,Downton Boom"'14 genannt werden. Darüber hinaus wird die Dissertation

\footnotetext{
Martin Lücke/Irmgard Zündorf, Einführung in die Public History, Göttingen 2018, S. 25.

Andreas Sommer, Geschichtsbilder und Spielfilme. Eine qualitative Studie zur Kohärenz zwischen Geschichtsbild und historischem Spielfilm bei Geschichtsstudierenden (Geschichtskultur und historisches Lernen 5), Berlin 2010, S. 15.

Korte/Paletschek, Geschichte in populären Medien, S. 32.

6 Andrew Higson, Nostalgia is not what it used to be. Heritage films, nostalgia websites and contemporary consumers, in: Consumption Markets \& Culture 17 (2014), Heft 2, S. 120-142, hier S. 123.

Definitionen der Begriffe Public History und Filmtourismus siehe S. 11.

David Lowenthal, Why the Past Matters, in: Heritage \& Society 4 (2011), Heft 2, S. 159-171, hier S. 159.

Definition des Begriffs Szenische Historienserie siehe S. 5.

10 Lücke/Zündorf, Einführung in die Public History.

11 Albrecht Steinecke, Filmtourismus, Konstanz-München 2016.

12 Andrew Higson, English heritage, English cinema. Costume drama since 1980, Oxford 2003; Andrew Hickson, Film England. Culturally English filmmaking since the 1990s, London-New York 2011.

13 J. P. Sperati/Sabine Schreiner, Downton Abbey on Location. An unofficial Review \& Guide to the Locations used, Sawston-Cambridge 2017.

14 Oliver Cox, The "Downton Boom". Country Houses, Popular Culture, and Curatorial Culture, in: The Public Historian 37 (2015), Heft 2, S. 112-119.
} 
von Jorden Hellemans, welcher die touristischen Folgen von Fernsehserien untersucht, herangezogen. ${ }^{15}$

Während das erste der drei Hauptkapitel auf das Verhältnis der Geschichtswissenschaft zum Film, insbesondere zu den Szenischen Historienserien, eingeht, widmet sich der zweite Abschnitt im Speziellen dem British Costume Drama und der Serie „Downton Abbey". Der dritte Teil beschreibt die Auswirkung der Fernsehserie auf ausgewählte Orte. In einem abschließenden Fazit werden die Ergebnisse zusammenfassend dargestellt.

\section{Ein ungleiches Paar - Die Geschichtswissenschaft und Szenische Histo- rienserien}

Traditionell beschäftigte sich die Geschichtswissenschaft vor allem mit Texten. ${ }^{16}$ So geht Volker Sellin in seiner "Einführung in die Geschichtswissenschaft" noch im Jahr 2005 davon aus, dass schriftliche Zeugnisse jeglicher Art die wichtigsten Quellen darstellen. ${ }^{17}$ Das Potenzial anderer Quellengattungen wurde lange ignoriert, erst in den 1970erJahren mehrten sich Forderungen, Bilder als Quellen in historische Fragestellungen miteinzubeziehen. In den 1980er-Jahren plädierten Alltags- und Sozialhistoriker*innen zudem für die Anerkennung des Films als Quelle in der Geschichtswissenschaft. ${ }^{18}$ Das in den 1990er-Jahren von Jan und Aleida Assmann weiterentwickelte Konzept des kollektiven Gedächtnisses geht davon aus, dass die Erinnerung jedes Individuums vom Kollektiv geprägt wird. ${ }^{19}$ Das Konzept verweist dabei auch auf die wichtige Rolle von Medien bei der Erinnerung an die Vergangenheit. ${ }^{20}$ Die Geschichtsvermittlung muss zwar nicht intentional erfolgen, ${ }^{21}$ die Tradierung von Vergangenem - ob absichtlich oder nicht - hat aber wesentliche Auswirkungen auf das Geschichtsbild, also auf die Vorstellung der Vergangenheit. Medien prägen somit die kollektive Erinnerung und dienen nicht nur der Darstellung, sondern ebenso der Wissensproduktion. ${ }^{22}$ Allen voran gilt der Spielfilm als "geschichtsbewusstseinskonstituierendes Medium".23

Die Wirkungsmacht von Bildern erkannte etwa John Thomas Mitchell und prägte 1994 den Begriff pictorial turn, der sich ebenso auf die Geschichtswissenschaft auswirkte. ${ }^{24} \mathrm{Da}$ das Fernsehen in den 1990er-Jahren zunehmend zu einem Leitmedium der populären

15 Jorden Hellemans, Film Induced Tourism. Television Series, Diss. Universidad de Murcia 2014

16 Sommer, Geschichtsbilder, S. 19.

17 Volker Sellin, Einführung in die Geschichtswissenschaft, Göttingen-Oxford 2009, S. 85.

18 Gerhard Paul, Von der historischen Bildkunde zur Visual History. Eine Einführung, in: Gerhard Paul (Hrsg.), Visual History. Ein Studienbuch, Göttingen 2006, S. 7-36, hier S. 8-9.

19 Aleida Assmann/Jan Assmann, Das Gestern im Heute. Medien und soziales Gedächtnis, in: Klaus Merten/ Siegfried Schmidt/Siegfried Weischenberger (Hrsg.), Die Wirklichkeit der Medien. Eine Einführung in die Kommunikationswissenschaft, Opladen 1994, S. 114-140, hier S. 117.

20 Assmann/Assmann, Das Gestern im Heute, S. 120; Jan Assmann, Das kulturelle Gedächtnis. Schrift, Erinnerung und politische Identität in frühen Hochkulturen, München 2018 ${ }^{8}$, S. 35-37.

21 Harald Welzer, Das soziale Gedächtnis, in: Harald Welzer (Hrsg.), Das soziale Gedächtnis. Geschichte, Erinnerung, Tradierung, Hamburg 2001, S. 11-14.

22 Korte/Paletschek, Geschichte in populären Medien, S. 15.

23 Sommer, Geschichtsbilder, S. 11.

24 Ebd., S. 20 
Vergangenheitsvermittlung avancierte, ${ }^{25}$ musste die Geschichtswissenschaft notgedrungen ihr Verhältnis zum Spielfilm ${ }^{26}$ als Medium der Geschichtsdarstellung ändern. Schrittweise etablierte sich die Visual History, welche als „interdisziplinärer Zugang zur visuellen Darstellung von Vergangenheit" ${ }^{\prime 27}$ zusammengefasst werden kann. Allerdings herrscht in der Geschichtswissenschaft nach wie vor Skepsis gegenüber der Komplexität des Films. Ein grundsätzliches Problem stellen die Fiktionalität und die Frage der Authentizität dar. ${ }^{28}$ Vergangenes Geschehen wird durch den Fokus auf einige wenige Hauptdarsteller*innen personalisiert ${ }^{29}$ und Emotionen werden bewusst eingesetzt, da dramatische Ereignisse besonders in Erinnerung bleiben. ${ }^{30}$ Die Intention dahinter ist klar, es geht in erster Linie nicht um eine möglichst seriöse Geschichtsdarstellung, sondern um Spannung und Unterhaltung - und somit um Quoten und Geld. ${ }^{31}$

Die vorliegende Arbeit beschäftigt sich mit den sogenannten Szenischen Historienfilmen. Dabei handelt es sich um Filme, die außerhalb des ungefähr achtzig bis hundert Jahre zurückreichenden Erinnerungs- und Erfahrungshorizonts des Publikums liegen. Die Handlung spielt in der Vergangenheit und wird unmittelbar durch Schauspieler*innen - also durch szenisches Spiel - dargestellt.32 Eine besondere Form innerhalb dieser Gattung stellen Szenische Historienserien dar, die durch ihren wiederholenden Charakter in Form von zahlreichen Folgen sowie meist mehreren Staffeln eine längere Laufzeit als klassische Spielfilme haben. ${ }^{33}$ Mit einem offenen Ausgang am Ende jeder Episode, einem Cliffhanger, wird Spannung aufgebaut und versucht, die Zuseher*innen langfristig an die Handlung zu binden. ${ }^{34}$

\section{British Costume Drama und die Fernsehserie „Downton Abbey“}

Wie das 452 Seiten umfassende Buch "The British Cinema Book" verdeutlicht, ist die britische Filmgeschichte äußerst facettenreich. ${ }^{35} \mathrm{Im}$ Zuge des Heritage Booms, der in Großbritannien im letzten Drittel des 20. Jahrhunderts einsetzte und eine verstärkte Bezugnahme auf das kulturelle sowie nationale Erbe bewirkte, ${ }^{36}$ entwickelte sich zunehmend ein Trend hin zu Spielfilmen mit historischen Inhalten. ${ }^{37}$ So spielen zwölf Prozent der zwischen 1990 und 2009 gedrehten britischen Spielfilme in der Vergangenheit. Von diesen rund 220 Produktionen waren 120 Filme in der Zeitspanne von

25 Fischer/Schuhbauer, Geschichte in Film, S 9.

26 Zwar sind auch Geschichtsdokumentationen ein Medium der Geschichtsdarstellung, die vorliegende Seminararbeit beschränkt sich bei ihren Ausführungen jedoch auf Spielfilme bzw. im Speziellen auf Szenische Historienserien.

27 Sommer, Geschichtsbilder, S. 25.

28 Ebd., S. 31.

29 Ebd., S. 30

30 Fischer/Schuhbauer, Geschichte in Film, S. 19.

31 Ebd., S. 100-101.

32 Fischer/Schuhbauer, Geschichte in Film, S. 40-41.

33 Eine Ausnahme stellen mehrteilige Spielfilme dar.

34 Steinecke, Filmtourismus, S. 181; Sue Beeton, Film-Induced Tourism (Aspects of Tourism 76), Clevedon 2016, S. 13.

35 Robert Murphy (Hrsg.), The British Cinema Book, London 2018.

36 Korte/Paletschek, Geschichte in populären Medien, S. 10.

37 Higson, Film England, S. 191. 
1800 bis 1939 angesiedelt, womit es sich um Szenische Historienfilme handelt. ${ }^{38}$ Diese Hinwendung zur Geschichte wird mit dem rasanten gesellschaftlichen Wandel in den letzten Jahrzehnten begründet:

„Traditionelle Wertemuster, Lebensstile und Arbeitsweisen waren [...] radikalen Veränderungen unterworfen; dies gilt auch für staatliche Grenzen und Konstruktionen von Nationen und Ethnien. Kategorien sozialer Ungleichheit wie Geschlecht, Klasse, Rasse oder Alter erfuhren einen teilweise rapiden Wandel ihrer Bedeutung für die gesellschaftliche Platzierung und Identität des Individuums. Die Hinwendung zur Geschichte kann in dieser Situation Kontinuität, Identität und Orientierung stiften." ${ }^{\prime 39}$

Für Großbritannien trifft diese Einschätzung wohl in besonderem Ausmaß zu. So ist es nicht verwunderlich, dass sich gerade dort ein neues Filmgenre herausbildete, welches auf das einstige britische Weltreich Bezug nimmt. ${ }^{40}$ Die Rede ist vom sogenannten British Costume Drama, das sich auf vielfältige Weise mit der britischen Geschichte und ihren Traditionen auseinandersetzt. ${ }^{41}$ Der Fokus dieser meist im späten 19. und frühen 20. Jahrhundert angesiedelten Filme liegt hauptsächlich auf den Privilegierten, Reichen und Adeligen. ${ }^{42}$ Romantische Komödien und Dramen ${ }^{43}$ vermitteln ein Bild von Großbritannien, insbesondere von England, das vor allem die oberen Gesellschaftsschichten in Szene setzt:

„Many of the films include set-piece celebratory events, lavish dinner parties or balls, for instance, which provide plenty of opportunities for filling the frame with splendid costumes and hair-dos, tableware and food. Equally frequently, conversations take place against a backdrop of picturesque semi-rural English scenery, or the frontage of some magnificent castle, stately home, or quaint cottage, the types of ancient architectural and landscape properties conserved by the National Trust and English Heritage."44

Diese Charakteristika treffen beispielsweise auf Spielfilme wie "Wings of the Dove" (1997), "An Ideal Husband" (1999) oder "The Importance of Being Earnest" (2002) zu, aber auch verschiedene Serien können dem British Costume Drama zugeordnet werden. ${ }^{45}$ Zu nennen wären hier unter anderem die bereits in den 1960er-Jahren gedrehte Serie „The Forsyte Saga" (1967-1969) sowie „Upstairs, Downstairs" (1971-1975) und „Bri-

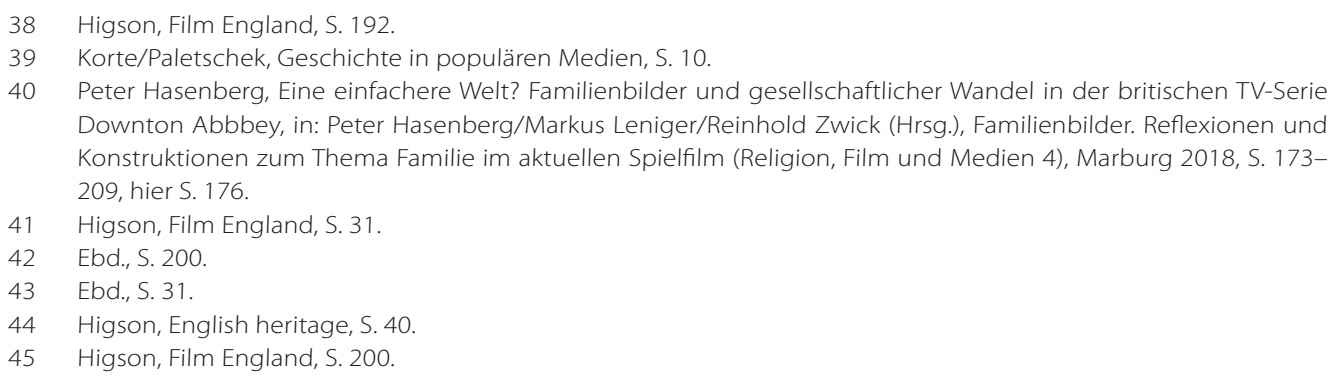


deshead Revisited" (1981). Eine neuere Produktion, welche durch die Thematisierung einer Adelsfamilie und ihrer Dienerschaft bestimmte Aspekte der vorherigen Serien aufgreift, stellt „Downton Abbey" (2010-2015) dar. ${ }^{46}$

An diesen Fernsehsendungen beanstanden Kritiker*innen vor allem die Konstruktion einer nationalen Identität, welche sich vorwiegend auf die upper-class bezieht. ${ }^{47}$ Wie Andrew Higson betont, handle es sich um eine idealisierte Version der britischen Geschichte, ${ }^{48}$ welche den Anschein erwecke, die oberste Gesellschaftsschicht sei das wichtigste Element des nationalen Erbes. ${ }^{49}$ Seiner Ansicht nach verbreiten diese "films of dubious national identity"50 nur eine begrenzte Darstellung der multikulturellen Gesellschaft..$^{51}$ Sie fokussieren sich auf "a highly circumscribed set of traditions, those of the privileged, white, Anglo-Saxon community who inhabit lavish properties in a semi-rural southern England, within striking distance of the metropolitan seat of power." ${ }^{\prime 52}$

Nichtsdestotrotz prägen diese Filme das kollektive Gedächtnis hinsichtlich der britischen Geschichte und Kultur entscheidend. ${ }^{53}$ Auch die britische Regierung erkannte diesen Umstand und erklärte: „British films are an important part of our cultural heritage and a significant channel for the continuing expression and dissemination of British culture." ${ }^{44}$ Für das British Costume Drama trifft dies besonders zu, gilt es doch als „the most British of all television genres ${ }^{\prime 55}$. Doch die Bezeichnung British ist trügerisch. Britishness spielt zwar eine Schlüsselrolle in der Festlegung und Reproduzierung des Markenzeichens Großbritanniens ${ }^{56}$, paradoxerweise wird diese aber oft von ausländischen Filmemacher*innen und Schauspieler*innen verkörpert. ${ }^{57}$ Dem Boom des British Costume Drama tut das aber keinen Abbruch - im Gegenteil. Gerade die Tourismus-Industrie profitiert davon, da viele Fans wegen solcher Filme nach Großbritannien reisen. ${ }^{58}$

Die vorliegende Seminararbeit möchte die Effekte, welche ein British Costume Drama auf den Tourismus und die öffentliche Geschichtsdarstellung haben kann, am Beispiel der Serie "Downton Abbey"59 aufzeigen. Es handelt sich dabei um eine vom britischen Sender ITV in Zusammenarbeit mit Carnival Films produzierte Historienserie, die von

\footnotetext{
46 Hasenberg, Eine einfachere Welt?, S. 177.

47 Claire Monk, The British heritage-film debate revisited, in: Claire Monk/Amy Sargeant (Hrsg.), British historical cinema. The history, heritage and costume film (British popular cinema), London-New York 2002, S. 176-198, hier S. 179 .

48 Higson, Film England, S. 208.

49 Higson, English heritage, S. 27.

50 Ebd., S. 26.

51 Higson, Film England, S. 31-32.

52 Higson, English heritage, S. 27.

53 Ebd., S. 1.

54 HM Treasury (Hrsg.), Reform of film taxes incentives. Promoting the sustainable production of culturally British films, Juli 2005, www.hm-treasury.gov.uk/d/filmcondocv1.pdf, eingesehen 18.3.2009, zit. nach Higson, Film England, S. 247.

55 Jerome de Groot, Foreword, in: James Legott/Julie Anne Taddeo (Hrsg.), Upstairs and Downstairs. British Costume Drama Television from The Forsyte Saga to Downton Abbey, London 2015, S. IX-XII, hier S. IX.

56 Higson, Film England, S. 191.

57 Ebd., S. 247.

58 Higson, English heritage, S. 56-63.

59 Michael Engler, Downton Abbey, DVD, 2960 min., London 2010-2015. Diese Arbeit beschränkt sich ausschließlich auf die Serie „Downton Abbey", der 2019 erschienene Spielfilm ist nicht Teil der Untersuchung.
} 
2010 bis 2015 im britischen und ein Jahr zeitversetzt im deutschsprachigen Fernsehen ausgestrahlt wurde. ${ }^{60}$ Die Serie thematisiert in sechs Staffeln und 52 Folgen das Leben der englischen Adelsfamilie Crawley und ihrer Dienstboten von 1912 bis 1926. ${ }^{61}$ Die Familie und das Personal, welche auf dem titelgebenden Anwesen Downton Abbey in der englischen Grafschaft Yorkshire leben, werden im Verlauf der Serie mit den gesellschaftlichen und politischen Veränderungen sowie mit den technischen Neuerungen des beginnenden 20. Jahrhunderts konfrontiert. So spielen etwa der Untergang der Titanic, der Erste Weltkrieg, die Spanische Grippe, die Emanzipation der Frau und die zunehmende Motorisierung eine Rolle. Zentrales Hauptthema ist jedoch der Erhalt des Anwesens samt Vermögen und die Frage nach dem legitimen Erben. Robert Crawley, Earl of Grantham und Verwalter von Downton, hat zwar drei Töchter, aufgrund der gesetzlichen Bestimmungen können diese das Erbe aber nicht antreten. Somit wird versucht, eine andere Lösung zu finden und die Töchter, insbesondere die Älteste, mit passenden Kandidaten zu verheiraten. Da die jungen Frauen allerdings eigene Pläne verfolgen und sich zunehmend emanzipieren, gestaltet sich dies als schwierig. Insgesamt scheitert der Versuch, den Status Quo aufrecht zu erhalten, in den meisten Fällen am unaufhaltsamen gesellschaftlichen Wandel.

Jane Mattisson bezeichnet "Dowton Abbey" als die erfolgreichste TV-Serie in der britischen Fernsehgeschichte ${ }^{62}$ und Peter Hasenberg stellt die These auf, dass keine andere Serie das öffentliche Bewusstsein und die Populärkultur in ähnlicher Art geprägt habe. ${ }^{63}$ Die Autorin der vorliegenden Arbeit möchte sich diesen Superlativen, die meist auf individueller Wahrnehmung beruhen, nicht anschließen, da dabei die Frage der Beweisbarkeit offen bleibt. Fest steht aber, dass „Dowton Abbey“ auf viele Menschen eine große Faszination ausübt. So wurde die erste Folge im britischen Fernsehen bereits von neun Millionen Personen gesehen, was einem Marktanteil von 32 Prozent entsprach. ${ }^{64}$ Das Finale der dritten Staffel verfolgten in Großbritannien zwölf Millionen Menschen im Fernsehen. ${ }^{65}$ Weltweit wird das Publikum der gesamten Serie auf etwa 120 Millionen Zuseher*innen geschätzt. ${ }^{66}$

Trotz zahlreicher Auszeichnungen auf nationaler und internationaler Ebene fallen die Kritiken zu "Downton Abbey" sehr unterschiedlich aus. Während beispielsweise der Filmkritiker Hasenberg die Serie positiv beurteilt und die universelle Botschaft des gesellschaftlichen Wandels betont, ${ }^{67}$ sind andere Beiträge vermehrt negativ konnotiert. So schreibt die „Süddeutsche Zeitung", es handle sich um „die unkritischste Darstellung

60 Hellemans, Film Induced Tourism, S. 42.

61 Hasenberg, Eine einfachere Welt?, S. 173.

62 Jane Mattisson, Downton Abbey. A Cultural Phenomenon. History for the Many, in: S/C. Journal of Literature, Culture and Literary Translation 5 (2014), S. 1-27, hier S. 1, https://www.sic-journal.org/Article/Index/291, eingesehen 1.5.2020.

63 Hasenberg, Eine einfachere Welt?, S. 177

64 Sperati/Schreiner, Downton Abbey, S. 5.

65 Lucy Brown, Homosexual Lives. Representation and Reinterpretation in Upstairs, Downstairs and Downton Abbey, in: James Legott/Julie Anne Taddeo (Hrsg.), Upstairs and Downstairs. British Costume Drama Television from The Forsyte Saga to Downton Abbey, London 2015, S. 263-273, hier S. 272.

67 Hasenberg, Eine einfachere Welt?, S. 209. 
des britischen Klassensystems"68. Die Zeitung „The Guardian“ schließt sich mit der Formulierung „idealised portrayals of aristocracy and social order"69 diesem Urteil weitgehend an. Der amerikanische Historiker Simon Schama bezeichnet die Serie sogar als "a steaming, silvered tureen of snobbery"70. Wie die Schlagzeile „Eine Nation flüchtet in die Vergangenheit" in der "Frankfurter Allgemeinen" ${ }^{\prime 71}$ oder der Artikel von Katherine Byrne $^{72}$ verdeutlichen, befürchten Kritiker*innen, dass ein Konservatismus mit klarer hierarchischer Ordnung vermittelt wird. James Legott, Professor für Film und Fernsehen, und Julie Anne Taddeo, Historikerin, Ioben „Downton Abbey“ zwar für die Detailtreue, bewerten die ihrer Ansicht nach nostalgische und konservative Verehrung des aristokratischen Lebensstils aber ebenso kritisch. ${ }^{73}$ Trotz der exemplarisch aufgezeigten Polarisierung der Serie legt die vorliegende Arbeit ihren Fokus jedoch nicht primär auf deren historische Authentizität, sondern thematisiert die Auswirkungen der Darstellung auf die Public History und den Filmtourismus.

\section{Auswirkungen auf Public History und Filmtourismus}

"It is time to [...] recognize its huge contribution to the British (and international) imaginative economy over decades. We may recognize its conservative impetus and worry at the nostalgic way it represents the past, but we should also recognize that it is something worth to investigate for its longevity, its influence, and its adaptability as a mode."74

Wie Jerome de Groot in Bezug auf das British Costume Drama feststellt, ist es an der Zeit, nicht nur die Art der Darstellung in Frage zu stellen, sondern auch auf ihre Effekte einzugehen. Im Fall von „Dowton Abbey“ haben sich bereits mehrere Publikationen mit dem Thema auseinandergesetzt. ${ }^{75}$ Die Autor*innen betonen dabei, dass die Rezeption der Serie zu einem regelrechten Massenphänomen geworden sei. ${ }^{76}$ So profitieren zahlreiche Branchen vom Hype um die Serie. Dies verdeutlichen beispielsweise die unzäh-

68 Christian Zaschke, Reich und schön, in: Süddeutsche Zeitung, 15.12.2017, https://www.sueddeutsche.de/medien/ das-empire-gastiert-in-new-york-reich-und-schoen-1.3793889? reduced=true, eingesehen 22.3.2020.

69 Tanya Gold, Downton Abbey. class and distinction, in: The Guardian, 13.9.2011, https://www.theguardian.com/ tv-and-radio/2011/sep/13/downton-abbey-class-and-distinction, eingesehen 22.3.2020.

70 Simon Schama, Why Americans have fallen for Snobby 'Downton Abbey', in: Newsweek, 16.1.2012, https://www. newsweek.com/why-americans-have-fallen-snobby-downton-abbey-64157, eingesehen 22.3.2020.

71 Gina Thomas, Eine Nation flüchtet in die Vergangenheit, in: Frankfurter Allgemeine, 22.9.2013, https:// www.faz.net/aktuell/feuilleton/medien/fernsehserie-downton-abbey-iv-eine-nation-fluechtet-in-dievergangenheit-12583246.html, eingesehen 22.3.2020.

72 Katherine Byrne, New Developments in Heritage. The Recent Dark Site of Downton "Downer" Abbey, in: James Legott/Julie Anne Taddeo (Hrsg.), Upstairs and Downstairs. British Costume Drama Television from The Forsyte Saga to Downton Abbey, London 2015, S. 177-189.

73 James Legott/Julie Anne Taddeo, Indroduction, in: James Legott/Julie Anne Taddeo (Hrsg.), Upstairs and Downstairs. British Costume Drama Television from The Forsyte Saga to Downton Abbey, London 2015, S. XVXXX, hier S. XXV.

74 Groot, Foreword, S. XII.

75 Mattisson, Downton Abbey; Oliver Cox, The "Downton Boom". Country Houses, Popular Culture, and Curatorial Culture, in: The Public Historian 37 (2015), Heft 2, S. 112-119.

76 Byrne, New Developments, S. 178. 
ligen auf „Downton Abbey" bezogenen Bücher, Spiele und Kostüme. ${ }^{77}$ Byrne spricht in diesem Zusammenhang von einer Kommerzialisierung der Vergangenheit ${ }^{78}$ und auch der Titel der vorliegenden Seminararbeit - Downton-Mania - erscheint angesichts dieser Entwicklung passend.

Eine der größten Auswirkungen hat der Downton Effect ${ }^{79}$ auf die Tourismusindustrie. ${ }^{80}$ Wie Albrecht Steinecke in seiner Monographie „Filmtourismus" betont, sind Spielfilme und TV-Serien entscheidend an der Imagebildung von Destinationen und den damit einhergehenden touristischen Effekten beteiligt. ${ }^{81}$ Dies bestätigt die nationale Tourismusagentur VisitBritain:

"Films and TV are powerful motivators for travel with almost a third of potential visitors to the UK wanting to visit places seen-on-screen. [...] The success of British period TV drama [British Costume Drama] [...] have also delivered a real boost to visitor numbers, including to buildings used as filming locations and showcasing less explored regions."12

Dieses Phänomen trifft ebenfalls auf „Downton Abbey“ zu. Laut Mattison beeinflusst die Serie das kollektive Gedächtnis der Zuschauer*innen hinsichtlich der britischen Vergangenheit ${ }^{83}$ - eine nicht unumstrittene Ansicht. Stephen Heath etwa argumentiert dagegen, das Fernsehen produziere Vergessen. ${ }^{84}$ In den meisten Fällen wird beim Publikum aber der Wunsch erweckt, die Drehorte zu besuchen. ${ }^{85} \mathrm{Um}$ die Auswirkungen der Fernsehserie „Downton Abbey" auf die Public History und den Filmtourismus untersuchen zu können, muss zunächst die Terminologie geklärt werden. Public History ist jede Form von Geschichtspräsentation für eine nicht geschichtswissenschaftlich vorgebildete Öffentlichkeit sowie eine Teildisziplin der Geschichtswissenschaft, die sich mit der Erforschung von Geschichtsdarstellungen befasst. ${ }^{86}$ Der Begriff Filmtourismus umfasst hingegen Reisen zu Drehorten und Schauplätzen von Spielfilmen und TV-Serien, den sogenannten On Locations. Zudem zählen Besuche von filmbezogenen Freizeiteinrichtungen bzw. Events dazu, welche allgemein OffLocations genannt werden. ${ }^{87}$ Die nachfolgenden Unterkapitel beschäftigen sich mit ausgewählten Orten dieser Art.

77 Nancy West, The Worlds of Downton Abbey, in: South Atlantic Review 80 (2015), Heft 3, S. 215-233, hier S. 215; Byrne, New Developments, S. 177.

78 Ebd. S. 178.

79 Cox, The 'Downton Boom', S. 115.

80 Hellemans, Film Induced Tourism; Lorenzo Bagnoli, Downton Abbey“" and the TVinduced Tourism, in: AlmaTourism. Journal of Tourism, Culture and Territorial Development 4 (2015), S. 102-116.

81 Steinecke, Filmtourismus, S. 5.

82 VisitBritain (Hrsg.), Global partnership campaigns, O. D., https://www.visitbritain.org/global-partnershipcampaigns, eingesehen 24.3.2020.

83 Mattisson, Downton Abbey, S. 1.

84 Stephen Heath, Representing Television, in: Patricia Mellankamp (Hrsg.), Logics of Television. Essays in Cultural Criticism, Bloomington 1990.

85 Hasenberg, Eine einfachere Welt?, S. 176

86 Lücke/Zündorf, Einführung in die Public History, S. 24.

87 Steinecke, Filmtourismus, S. 45. 


\subsection{On Locations}

Wie das Buch „Downton Abbey on Location“88 verdeutlicht, entstand die Serie an den unterschiedlichsten Orten in Großbritannien. Im Folgenden werden zwei für die Serie bedeutsame On Locations herausgegriffen und näher beschrieben.

\subsubsection{Highclere Castle}

Das im Verwaltungsbezirk Hampshire, südwestlich von London gelegene Highclere Castle stellt das Anwesen Downton Abbey dar und ist somit als Familiensitz der Crawleys der Hauptfilmort der Serie. Alle Szenen in den Räumlichkeiten der Familie sowie die Außenaufnahmen im Park wurden dort gedreht. Auch wenn sich das Herrenhaus auf einem Grundstück befindet, das die Bischöfe von Winchester bereits im Mittelalter genutzt haben, erhielt das Gebäude sein heutiges Aussehen erst im 19. Jahrhundert und wurde im neogotischen Stil zwischen 1839 und 1842 errichtet. Heute bewohnt der achte Earl of Carnarvon mit seiner Familie das Schloss. ${ }^{89}$

Zwar spielte das Anwesen in mehreren Produktionen, wie „The Secret Garden" (1987), "Robin Hood Prince of Thieves" (1991) oder "The Four Feathers" (2002) eine Rolle, gröBere Bekanntheit erlangte es aber vor allem durch „Downton Abbey“.90 Während sich der Earl und die Countess of Carnarvon vor dem Seriendreh noch in ernsthaften Zahlungsschwierigkeiten befanden und das renovierungsbedürftige Highclere Castle kaum erhalten konnten, erlebte das Schloss nach der Ausstrahlung der Fernsehserie einen regelrechten Aufschwung. In der Sommersaison 2010 - also bevor „Downton Abbey" im Herbst desselben Jahres erstmals ausgestrahlt wurde - verzeichnete es 30.000 Gäste. 2011 - nach dem Serienstart - besuchten 50.000 Personen das Schloss ${ }^{91}$ und 2014 stieg die Zahl auf 60.000. ${ }^{92}$ Im Durchschnitt besichtigen Highclere Castle in den Sommermonaten ungefähr 1.200 Menschen pro Tag. ${ }^{93}$

Um den Andrang der Besucher*innen zu kontrollieren und sicherzustellen, dass sich nicht zu viele Menschen gleichzeitig auf dem Gelände aufhalten, war der Besitzer gezwungen, ein timed ticket system einzuführen ${ }^{94}$ : Die Tickets können seitdem nur im Voraus über die Webseite von Highclere Castle gekauft werden und sind nur noch in begrenzter Anzahl verfügbar. Aufgrund der enormen Nachfrage und der Tatsache, dass das Anwesen ausschließlich im Sommer besucht werden kann, war beispielsweise bereits am 4. April 2020 kein einziges Ticket mehr für den Juli 2020 erhältlich. ${ }^{95}$

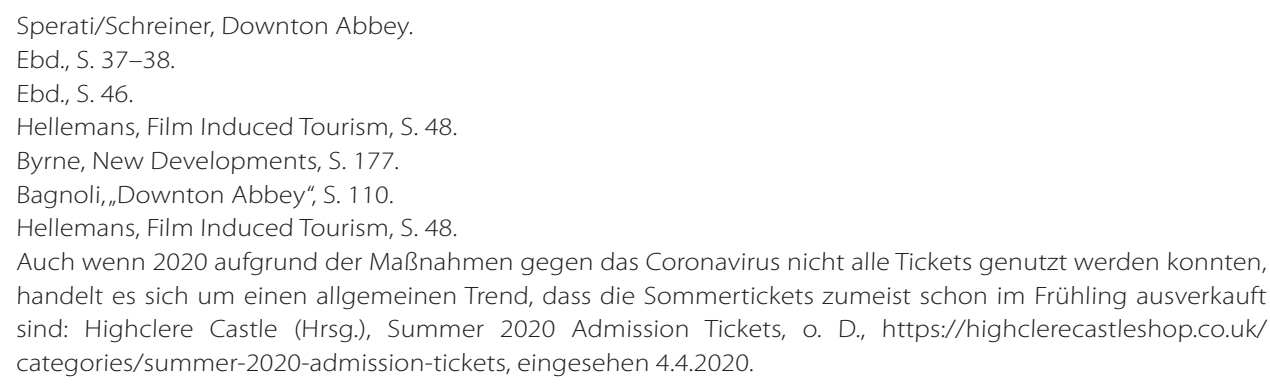


Personen, welche das Schloss besichtigen möchten, müssen Monate vorher einen Tag festlegen, an dem ihr Ticket für einige wenige Stunden Gültigkeit besitzt. Zudem sind spezielle Veranstaltungen buchbar. Die Countess of Carnarvon bietet, in Anlehnung an „Downton Abbey", die persönlich geführte „Living in a Castle Tour"96 an:

"Following each talk, which will take place in the Saloon, the heart of the Castle, guests have the chance to enjoy a guided Tour both to explore the works of art, but also the rooms which are, of course, the set in which 'Downton Abbey' was filmed [...]."97

Neben den Schlossbesichtigungen ist es in den Sommermonaten möglich, in verschiedenen Lodges zu übernachten. ${ }^{98}$ Einmalig wurde im Oktober 2019 eine Übernachtung für zwei Personen im Schloss angeboten, aufgrund der hohen Nachfrage gab es aber strenge Auswahlkriterien. Die Buchung glich einem Bewerbungsverfahren, bei dem die Interessent*innen ihre Leidenschaft für "Downton Abbey" überzeugend darlegen mussten. ${ }^{99}$ Wie beispielsweise die aufwendigen Renovierungen der um 1840 errichteten London Lodge und der ähnlich alten Grotto Lodge zeigen, ${ }^{100}$ scheint der Besitzer mittlerweile keine Probleme mehr mit den Erhaltungskosten zu haben. Bereits 2013 bestätigte der Earl of Carnarvon den durch „Downton Abbey“ hervorgerufenen Erfolg von Highclere Castle, welcher den finanziellen Druck verringert und wieder mehr Handlungsspielraum für dringend notwendige Investitionen geboten habe. ${ }^{101}$ Die langfristigen Auswirkungen der Serie trugen somit zum Erhalt des Gebäudes bei.

Der Earl und die Countess of Carnarvon investierten darüber hinaus in einen repräsentativen Internetauftritt. So gibt es neben der eigentlichen Homepage für das Schloss ${ }^{102}$ noch eine eigene Webseite für die Countess of Carnarvon. ${ }^{103}$ Anhand von Videos und einem Blog bietet die Gräfin Hintergrundinformationen zum Leben auf dem Schloss und dem Seriendreh an:

"My blog is my way of trying to share the stories and heritage of this wonderful building and estate, and all the people and animals that live and work here, so

96 Highclere Castle (Hrsg.), Summer 2020 Admission Tickets.

97 Highclere Castle (Hrsg.), Living in a Castle, o. D., https://highclerecastleshop.co.uk/categories/living-in-a-castle, eingesehen 4.4.2020.

98 Hellemans, Film Induced Tourism, S. 48.

99 Silvia Ihring, Ein Zimmer in „Downton Abbey“ kann man jetzt auf Airbnb mieten, in: Welt, 1.10.2019, https://www. welt.de/icon/unterwegs/article201175490/Airbnb-bietet-Uebernachtung-im-Schloss-aus-Downton-Abbey. html, eingesehen 4.4.2020.

100 Highclere Castle (Hrsg.), London Lodge, o. D., https://www.highclerecastle.co.uk/london-lodge, eingesehen 4.4.2020; Highclere Castle (Hrsg.), Grotto Lodge, o. D., https://www.highclerecastle.co.uk/grotto-lodge, eingesehen 4.4.2020.

101 Hellemans, Film Induced Tourism, S. 48; Viv Micklefield, Lady Carnarvon on Downton Abbey, life at Highclere Castle and her blog, in: Hampshire Life, 15.1.2018, https://www.hampshire-life.co.uk/people/lady-carnarvon-ondownton-abbey-life-at-highclere-castle-and-her-blog-1-5355878, eingesehen 4.4.2020.

102 Highclere Castle (Hrsg.), Home, o. D., https://www.highclerecastle.co.uk/, eingesehen 4.4.2020

103 Lady Carnarvon (Hrsg.), The official Website of the Countess of Carnarvon, o. D, https://www.ladycarnarvon.com/, eingesehen 4.4.2020. 
that you can get to know and love it as I do. Surrounded by so much history, I try to turn the stories into ways of drawing people into Highclere's world."104

Die wöchentlichen Blogeinträge erfreuen sich großer Beliebtheit und erreichen ein internationales Publikum. ${ }^{105}$ Je nach Thema erhalten sie etwa fünfzig bis 350 Kommentare. Besonders häufig werden die Eintragungen in der Rubrik "Downton Abbey“ kommentiert. Die Reaktionen fallen dabei durchwegs positiv aus und loben zum Beispiel Lady Carnarvon für ihr Engagement, schwärmen über die Serie oder berichten über Besuche in Highclere Castle. ${ }^{106}$ Ähnlich verhält es sich in den sozialen Medien: Highclere Castle verfügt über einen eigenen YouTube-Kanal, dem 1.220 Abonennt*innen folgen, ${ }^{107}$ und ist auf Facebook, Twitter sowie auf Instagram vertreten. ${ }^{108}$ Zudem ist eine kostenpflichtige App für das Smartphone erhältlich, welche das Schloss und seine Geschichte präsentiert und eine Verbindung zu „Downton Abbey“ herstellt. Beispielsweise führt die Stimme von Carson, dem Butler in „Downton Abbey“, die Nutzer*innen der Anwendung durch ausgewählte Räume. Es werden Gegenstände aus dem Schloss vorgestellt und es ist möglich, Highclere Castle mittels einer 360-Grad-Ansicht virtuell zu besichtigen. ${ }^{109}$ Auf der offiziellen Webseite heißt es: „This app allows you to explore the history of the Castle, its rooms and treasures and to discover what it's like to live and work there."110

"Downton Abbey" wirkt sich jedoch nicht nur auf Highclere Castle aus, sondern steigert den Tourismus in der gesamten Region, die umgangssprachlich bereits als Downtonia bezeichnet wird. Einer Studie aus dem Jahr 2013 zufolge kommen ein Drittel der Gäste ausschließlich in die Gegend, um die Drehorte von "Downton Abbey" zu besuchen. ${ }^{11}$ Zahlreiche Reisebüros haben sich auf "Downton Abbey" spezialisiert und bieten entsprechende Touren an. ${ }^{112}$ So konnte etwa die von Highclere ungefähr acht Kilometer entfernte Stadt Newbury einen Nutzen daraus ziehen: Besuchten im Jahr 2010 20.450 Tagesgäste pro Woche die Kleinstadt, waren es im Jahr 2013 wöchentlich bereits 25.090. ${ }^{113}$ Es kann also in der gesamten Region ein gesteigertes öffentliches Interesse an den historischen Orten und an Public History verzeichnet werden. Ähnliche Entwicklungen gibt es ebenfalls im Ort Bampton - der nächsten On Location, mit der sich die vorliegende Arbeit auseinandersetzt.

104 Lady Carnarvon (Hrsg.), About, o. D, https://www.ladycarnarvon.com/about/, eingesehen 4.4.2020.

105 Tammy Hughes, Downton Abbey is open for business. Lord and Lady Carnarvon re-open Highclere Castle to the public, in: The Daily Mail, 21.1.2017, https://www.dailymail.co.uk/news/article-4142220/Downton-Abbey-setopen-public.html, eingesehen 5.4.2020.

106 Lady Carnarvon (Hrsg.), Blog, o. D, https://www.ladycarnarvon.com/blog/, eingesehen 5.4.2020.

107 Highclere Castle (Hrsg.), YouTube, O. D., https://www.youtube.com/channel/UCLbmDFj2sQV4n4R1QaSfZlg/ featured, eingesehen 5.4.2020.

108 Highclere Castle (Hrsg.), Facebook, O. D., https://www.facebook.com/HighclereCastleOfficial/, eingesehen 5.4.2020; Highclere Castle (Hrsg.), Twitter, O. D., https://twitter.com/HighclereCastle, eingesehen 5.4.2020; Highclere (Hrsg.), Instagram, O. D., https://www.instagram.com/highclere_castle/, eingesehen 5.4.2020.

109 Highclere Castle (Hrsg.), Highclere Castle launches New App on iTunes and GooglePlay, 5.1.2016, https://www. highclerecastle.co.uk/news/highclere-castle-launches-new-app-itunes-and-google-play, eingesehen 5.4.2020.

110 Ebd.

111 Hellemans, Film Induced Tourism, S. 54; Sperati/Schreiner, Downton Abbey, S. 45

112 Hellemans, Film Induced Tourism, S. 45-46.

113 Bagnoli, ,Downton Abbey“, S. 110 


\subsubsection{Bampton}

Das im Verwaltungsbezirk Oxfordshire, nordwestlich von London gelegene Bampton, ein Ort mit 2.500 Einwohner*innen, stellt in der Serie das Dorf Downton dar. Auch wenn Bampton mit seinem historischen Ortskern, insbesondere der gut erhaltenen St. Mary's Church und seiner grünen Hügellandschaft (Cotswolds) durchaus Attraktionen aufweist, war der Tourismus hier nie von großer Bedeutung. Nach der Ausstrahlung der Serie änderte sich dies allerdings. „Downton Abbey“-Themenreisen wandelten die Gemeinde in vielerlei Hinsicht: Während die Ortschaft vorher über kein Besucherzentrum verfügte und sich nur wenige Urlauber*innen in den Ort verirrten, ist in der örtlichen Bücherei nun eine Tourismusinformation eingerichtet, welche täglich von mehreren hundert Personen besucht wird. ${ }^{114}$ Neben einer kleinen Ausstellung zum Dreh in Bampton werden ebenfalls auf die Serie bezogene Souvenirs angeboten und 2013 waren in der West Ox Arts Gallery im alten Rathaus einige in „Downton Abbey“ getragene Kostüme ausgestellt. ${ }^{115}$

Die Hauptattraktion für die Besucher*innen ist sicherlich die Church View Street, da dort die meisten in Bampton spielenden Szenen gedreht wurden. ${ }^{116}$ So steht zum Beispiel die St. Mary's Church, die fast in jeder Folge zu sehen war, an dieser Straße. Neben Hintergrundaufnahmen spielte die Kirche vor allem bei den Hochzeiten und Beerdigungen eine tragende Rolle in der Serie. Am Kirchenportal sind Fotos angebracht, welche Einblicke hinter die Kulissen der Dreharbeiten zeigen. ${ }^{17}$ Zudem können am Friedhof die für die Serie angelegten Gräber besichtigt werden. ${ }^{118}$ Weitere beliebte Gebäude sind etwa das Churchgate House, das in der Serie das Haus von Isobel Crawley darstellte, oder die ehemalige Grammar School, in der heute die Bücherei, das Besucherzentrum sowie das lokale Archiv untergebracht sind und das in der Serie als Downton Cottage Hospital diente. ${ }^{119}$

Auch die nationale Tourismusagentur VisitBritain sowie die regionale Einrichtung in Oxfordshire bewerben auf ihren Homepages mittlerweile die Downton Abbey Film Locations und verweisen an mehreren Stellen auf Bampton. Eigene Downton Leaflets und Walking Maps informieren die Gäste über die verschiedenen Angebote. ${ }^{120}$ Auf der Webseite der Region ist zu lesen:

„This charming village will seem oddly familiar to Downton Abbey fans, doubling as the fictional Yorkshire village of Downton [...]! Many scenes, including some of Downton Abbey's key events, have been filmed in and around the

114 Bagnoli, „Downton Abbey", S. 106-110.

115 Andrea David, Downton Abbey, in: Filmtourismus. Die Welt der Drehorte, Blogbeitrag o. D., https://www. filmtourismus.de/downton-abbey/, eingesehen 10.4.2020.

116 Ebd.

117 Sperati/Schreiner, Downton Abbey, S. 153.

118 David, Downton Abbey.

119 Sperati/Schreiner, Downton Abbey, S. 154-155.

120 VisitBritain (Hrsg.), Bampton, o. D., https://www.visitbritain.com/de/de/bampton, eingesehen 10.4.2020; Oxfordshire Cotswolds Tourism (Hrsg.), Downton Abbey film locations, o. D., https://www.oxfordshirecotswolds. org/plan-your-visit/towns-and-villages/downton-abbey-film-locations, eingesehen 10.4.2020. 
church in Bampton, the main location for filming. The set locations for Downton Abbey have become stars in their own right and are as much a character in the series as any of the actors."121

Die vierteljährlich erscheinende Gemeindezeitschrift „Bampton Beam” etablierte sogar eine eigene Rubrik zu "Downton Abbey“.122 Die Bedeutung von "Downton Abbey" für den Ort lässt sich ebenfalls am Bampton Community Archive erkennen: Dieses 2002 gegründete Archiv dokumentiert die Geschichte von Bampton. Neben steinzeitlichen Funden und historischen Fotografien sammelt die Institution sämtliche Zeugnisse, welche an die Dreharbeiten von „Downton Abbey“ in Bampton erinnern. ${ }^{123}$ Darunter fallen etwa Fotos oder Zeitungsartikel, welche in digitalisierter Form auf der Webseite frei zugänglich sind. Laut dem Archiv handelt es sich dabei um wichtige Quellen für die Geschichte des Ortes. ${ }^{124}$ Darüber hinaus hat das Archiv das Projekt „The,Downton' Mile“ ins Leben gerufen, welches die Restaurierung der alten Grammar School - in der Serie als Krankenhaus zu sehen - zum Ziel hat. Die Idee dahinter ist, so viele Ein-Pfundmünzen zu sammeln, wie theoretisch benötigt würden, um die eine Meile lange Strecke der "Downton Abbey Location Tour" in Bampton zu bedecken. Insgesamt sollen so 71.000 Pfund zusammenkommen. Schauspieler*innen aus "Downton Abbey", darunter Hugh Bonneville, in der Serie als Robert Crawley zu sehen, werben in der Öffentlichkeit für diesen Plan. Die Serie wird zum Anlass genommen, das historische Gebäude zu renovieren und dort neben der Bücherei, dem Besucherzentrum und dem Archiv eine detaillierte Ausstellung zu den „Downton Abbey“ Dreharbeiten in Bampton unterzubringen. ${ }^{125}$

Wie Albert Steinecke und Sue Beeton in zwei Standardwerken zum Filmtourismus betonen, hat dieser auch negative Effekte - ebenso in Bampton. ${ }^{126}$ Nach der Ausstrahlung von "Downton Abbey" empfahlen Anlagenberater*innen Investitionen im Ort, wodurch neue Siedlungen entstanden und die Immobilienpreise stiegen. Die ansässige Bevölkerung steht diesen Entwicklungen zum Teil kritisch gegenüber und befürchtet, dass die Infrastruktur bald überlastet sein und der Ort seinen ursprünglichen Charme verlieren könnte. Die Society for the Protection of Bampton versucht dagegen anzukämpfen - allerdings mit mäßigem Erfolg. ${ }^{127}$

\subsection{OffLocations}

Neben den sogenannten On Locations, den eigentlichen Drehorten von „Downton Abbey", entwickelten sich auch Orte, die zwar keinen räumlichen Bezug, dafür aber einen

121 VisitBritain (Hrsg.), Bampton.

122 Bampton Beam (Hrsg.), Downton Abbey, o. D., http://www.bamptonbeam.co.uk/downton-abbey, eingesehen 10.4.2020.

123 Bampton Community Archive (Hrsg.), About us, o. D., https://www.bamptonarchive.org/the-bamptoncommunity-archive/, eingesehen 10.4.2020.

124 Bampton Community Archive (Hrsg.), Downton Abbey, o. D., https://atom.bamptonarchive.org/index.php/ downton-abbey, eingesehen 10.4.2020.

125 Bampton Community Archive (Hrsg.), The ,Downton' Mile, o. D., http://www.downtonmile.co.uk/, eingesehen 10.4.2020

126 Steinecke, Filmtourismus, S. 135-184; Beeton, Film-Induced Tourism., S. 114-170.

127 Hellemans, Film Induced Tourism, S. 48-49. 
thematischen Zusammenhang mit der Serie haben, zu touristischen Sehenswürdigkeiten. In den folgenden Unterkapiteln werden ausgewählte Off Locations exemplarisch beschrieben.

\subsection{1 "Downton Abbey" Ausstellungen}

Fans von "Downton Abbey" sind nicht nur an der Handlung und den Charakteren der Serie interessiert, sondern zeigen ebenso Begeisterung für verwendete Kostüme und Gegenstände. ${ }^{128}$ Ein Beispiel dafür ist die bereits erwähnte Ausstellung in der West Ox Arts Gallery in Bampton. ${ }^{129}$ Die Serie löste ein allgemeines Interesse an den Herrenhäusern des englischen Landadels - den sogenannten stately homes oder English country houses - aus. ${ }^{130}$ Richard Compton, Präsident der Historic Houses Association, betonte in einem Interview: „Downton has done us all a great service because it's reminding people about our heritage. It's bringing history to light."131 Einer Umfrage zufolge besuchte im Jahr 2013 einer von drei Touristen*innen in Großbritannien ein solches Herrenhaus. Der Historiker Oliver Cox begründet dies damit, dass es diesen repräsentativen Gebäuden besonders gut gelinge, eine Verbindung zwischen Vergangenheit und Gegenwart herzustellen. Die Geschichten der Menschen, welche diese Anwesen bewohn(t)en, berühren die Besucher*innen emotional und ermöglichen ein Hineinversetzen in das damalige Leben. ${ }^{132}$

Daher lag es nahe, die beiden Aspekte Kostüme und stately homes zu kombinieren und an Orten, an denen die Serie nie gedreht wurde, „Downton Abbey“ Ausstellungen einzurichten. Beispielsweise war im US-amerikanischen Winterthur Museum and Country Estate von März 2014 bis Jänner 2015 die Ausstellung „Costumes of Downton Abbey” zu sehen. ${ }^{133}$ In der serienbezogenen Kostümausstellung wurden vierzig ausgewählte Gewänder aus der Serie präsentiert und mit entsprechenden Fotos ergänzt. Laut Museumsmarketing ermögliche die Ausstellung den Besucher*innen, die Welt von "Downton Abbey" zu betreten und zu erfahren. ${ }^{134}$ Auch wenn Historiker*innen wohl skeptisch sein sollten, wenn damit geworben wird, eine historisch korrekte Welt betreten und selbst erfahren zu können, handelte es sich um ein sehr erfolgreiches Public History-Projekt und um die am häufigsten besuchte Ausstellung in Winterthur. ${ }^{135}$

Angesichts dieses Erfolgs ließen weitere Projekte nicht lange auf sich warten. So heißt eine weitere Ausstellung, welche verschiedene Kostüme aus der Serie "Downton Abbey“ zeigt, „Dressing Downton: Changing Fashion for Changing Times". Die Schau wur-

128 Hellemans, Film Induced Tourism, S. 46.

129 David, Downton Abbey.

130 Cox, The "Downton Boom", S. 113.

131 Zit. nach Robert Hardiman, Defending the real Downton Abbeys. Why Britain's stately homes are struggling, in: The Spectator, 9.3.2013, https://www.spectator.co.uk/article/defending-the-real-downton-abbeys, eingesehen 5.4.2020.

132 Cox, The "Downton Boom", S. 114

133 Winterthur Museum (Hrsg.), Costumes of Downton Abbey, O. D., http://www.winterthur.org/exhibitions-events/ exhibitions/past-exhibitions/costumes-of-downton-abbey/, eingesehen 13.4.2020.

134 Ebd

135 Laura Jacobs, Upstairs Timelessness, Downstairs Precision, in: The Wall Street Journal, 8.7.2014, http://online.wsj. com/articles/exhibition-review-upstairstimelessness-downstairs-precision-1404861153, eingesehen 13.4.2020. 
de im Februar 2015 im Biltmore Estate in North Carolina/USA eröffnet und war seitdem in verschiedenen US-Museen zu sehen - beispielsweise 2016 im Driehaus Museum in Chicago, welches für die dreimonatige Ausstellungsdauer rund 30.000 Personen erwartete. ${ }^{136}$ Die Sammlung umfasst 35 Gewänder - darunter sowohl Neuanfertigungen nach historischen Vorbildern als auch originale Kleidungsstücke bzw. Accessoires aus den 1910er- und 1920er-Jahren - welche alle in der Serie getragen wurden. ${ }^{137}$

Darüber hinaus gibt es noch das Public History-Projekt „Downton Abbey: The Exhibition“, welches neben Kostümen noch Filmkulissen von „Downton Abbey“ präsentiert. Auf der offiziellen Ausstellungsseite heißt es dazu:

"Downton Abbey: The Exhibition, based on the beloved television show, transports you to post-Edwardian England, where the characters and the iconic house come to life. You'll be immersed in the fascinating social history, culture, and some of the most memorable moments from the show's six-season run."138

Auch in diesem Fall handelt es sich um eine Wanderausstellung. ${ }^{139}$ Sie zeigt 58 Kostüme sowie sechs Filmkulissen und eine Vielzahl an Requisiten aus der Serie - von Büchern und Briefen bis hin zu Handschuhen und Halsketten. Zudem werden Szenen aus der Serie vorgeführt und 21 Charaktere vorgestellt. ${ }^{140}$ Wie bereits die vorherigen Ausstellungsorte nutzte das Biltmore Estate in North Carolina - ein Landsitz aus den 1890erJahren, ${ }^{141}$ der von November 2019 bis April 2020 ${ }^{142}$ die Ausstellung beherbergte, ${ }^{143}$ die mediale Aufmerksamkeit, um die Ähnlichkeiten des eigenen Anwesens mit „Downton Abbey" hervorzuheben. ${ }^{144}$ So werden auf der Webseite des Guts Aktivitäten beworben, welche in Zusammenhang mit der Ausstellung gebucht werden können und Einblicke in die Geschichte des Hauses ermöglichen. In Anlehnung an die Serie "Downton Abbey", die sowohl das Leben der Adeligen als auch die Welt der Dienerschaft zeigt, bietet das Biltmore Estate eine „Through The Servants' Eyes Tour“ mit Besichtigung der Dienstbotenbereiche an. ${ }^{145}$

136 Alexandra Whittaker, Downton Abbey Exhibit coming to Chicago, in: Medill Reports Chicago, 27.1.2016, https:// news.medill.northwestern.edu/chicago/downton-abbey-exhibit-coming-to-chicago/, eingesehen 13.4.2020.

137 Ebd.

138 Downton Abbey: The Exhibition (Hrsg.), Experience the history, the fashion and the house, o. D., https://www. downtonexhibition.com/, eingesehen 15.4.2020.

139 Downton Abbey: The Exhibition (Hrsg.), Experience.

140 Biltmore Estate (Hrsg.), Downton Abbey: The Exhibition by the Numbers, 12.11.2019, https://www.biltmore.com/ blog/downton-abbey-the-exhibition-by-the-numbers/, eingesehen 15.4.2020.

141 Biltmore Estate (Hrsg.), Estate History, O. D., https://www.biltmore.com/our-story/estate-history/, eingesehen 15.4.2020.

142 Im Zuge der Coronamaßnahmen wurde die Ausstellung allerdings am 26. März 2020 geschlossen: Biltmore Estate (Hrsg.), COVID-19: Health and Safety Changes, O. D., https://www.biltmore.com/coronavirus-covid-19-update/, eingesehen 15.4.2020.

143 Biltmore Estate (Hrsg.), Downton Abbey: The Exhibition now open at Biltmore, 8.11.2019, https://www.biltmore. $\mathrm{com} /$ news-release/downton-abbey-the-exhibition-now-open-at-biltmore/, eingesehen 15.4.2020.

144 Biltmore Estate (Hrsg.), Comparing Biltmore House to Downton Abbey, 18.8.2019, https://www.biltmore.com/ blog/comparing-biltmore-house-to-downton-abbey-2/, eingesehen 15.4.2020.

145 Biltmore Estate (Hrsg.), Top 5 Downton Abbey-Related Activities at Biltmore, 7.11.2019, https://www.biltmore. com/blog/top-5-downton-abbey-related-activities-at-biltmore/, eingesehen 15.4.2020. 
Cox betont, dass Szenische Historienserien, wie "Downton Abbey", das Öffentliche Interesse an der Vergangenheit wecken und die Art und Weise, wie die Vergangenheit konsumiert wird, verändern. Die beschriebenen Ausstellungen verdeutlichen, dass sich dies auf die Konzeption von Museen, insbesondere auf stately homes, auswirkt. ${ }^{146}$ In dieser Veränderung liegt Potential:

„Downton Abbey has reinforced the importance of bringing alive the country house, challenging academics and curators to explore how competing personal narratives can be used to explain the architectural container that shaped and determined the evolution of these stories. The country house now holds potent sway over the visiting public through the personal stories of those who lived upstairs and down." ${ }^{147}$

Nichtsdestotrotz wurden die erwähnten Ausstellungen teils stark kritisiert: So schreibt die "Süddeutsche Zeitung", die Ausstellung „Downton Abbey: The Exhibition" präsentiere ein "England, das es so nie gab"148. Weiters ist nicht selten von "retro-kitsch fantasia" oder von "Disneyfication" die Rede. ${ }^{149}$ So taucht der Vorwurf auf, es gehe nur darum, die Besucher*innen zu unterhalten, sodass es kaum noch einen Unterschied zu einem Vergnügungspark gebe. ${ }^{150}$ "Downton Abbey" hat allerdings keine komplett neue kuratorische Richtung hervorgebracht, ${ }^{151}$ sondern bereits bestehende Trends in der musealen Präsentation beschleunigt und somit das öffentliche Interesse an den country houses gesteigert. ${ }^{152}$

Auch wenn über die serienbezogenen Ausstellungen keine genauen Besucherzahlen veröffentlicht wurden und nicht generell angenommen werden kann, dass sich alle Besucher*innen genauer mit den Ausstellungen auseinandergesetzt haben, kann argumentiert werden, dass es der Serie gelungen ist, ein größeres Publikum zu erreichen und historische Themen zu vermitteln. Es gilt allerdings zu berücksichtigen, dass Filme und Serien stets eine gewisse Erwartungshaltung bei den Zuschauer*innen implizieren, die nicht immer der Realität entspricht. ${ }^{153}$ Das Fernsehen vermittelt den Eindruck, die Vergangenheit erlebbar zu machen, aber die dabei erschaffene Welt kann nicht eins zu eins besichtigt werden. ${ }^{154}$ Die Herausforderung für die Geschichtswissenschaft und die Kulturbranche besteht daher darin, "the quasi-historical shorthand of Downton"155 zu nutzen, um Besucher*innen in die country houses zu bringen und deren Geschichte

\footnotetext{
146 Cox, The "Downton Boom", S. 114.

147 Ebd., S. 118-119.

148 Zaschke, Reich und schön.

149 Stephen Adams, National Trust is 'Disney-fying' its country houses, say critics, in: The Telegraph, 29.5.2010, http:// www.telegraph.co.uk/culture/culturenews/7778870/NationalTrust-is-Disney-fying-its-country-houses-saycritics.html, eingesehen 18.4.2020.

150 Cox, The "Downton Boom", S. 119.

151 Die museale Darstellung vom Leben der Dienerschaft ist nicht neu, sondern wurde bereits in den 1970 er-Jahren gezeigt: Cox, The "Downton Boom", S. 116

153 Ebd., S. 117.

154 Higson, Nostalgia, S. 129

155 Cox, The "Downton Boom", S. 115.
} 
mit wissenschaftlicher Recherche gemäß der Public History für ein breites Publikum aufzubereiten. ${ }^{156}$

\subsubsection{Downton Expierence auf Dundas Castle}

Im Bereich der Public History steht in den letzten Jahren nicht mehr die Darstellung von Authentizität im Mittelpunkt, sondern vielmehr das (Nach-)Erleben. ${ }^{157}$ Die Historikerin Suzannah Lipscomb erklärt dies damit, dass die Menschen glauben, sich besser in vergangene Welten hineinversetzen zu können, wenn sie die Vergangenheit scheinbar selbst erlebt haben. ${ }^{158}$ An sich stellt das Erleben von Vergangenheit in der Gegenwart zwar einen Widerspruch dar, dennoch erfreut sich die sogenannte Living History wachsender Beliebtheit, da sie versucht, Geschichte möglichst authentisch nachzuspielen. ${ }^{159}$ Es geht darum, „Geschichte spannend und für die Lebenswelt der Rezipienten anschlussfähig"160 zu gestalten.

Fans der Serie konnten 2016 mit der „Downton Experience“ auf dem schottischen Dundas Castle das in „Downton Abbey“ gezeigte Leben bei einem Urlaub in die Vergangenheit nacherleben. Das Paket umfasste sowohl upstairs als auch downstairs Aktivitäten. Da die „Downton Experience” mittlerweile nicht mehr angeboten wird, finden sich keine Informationen auf der offiziellen Webseite von Dundas Castle. Allerdings haben zahlreiche Medien darüber berichtet, wobei die Preisangaben für einen Tag Aufenthalt zwischen 3.000 und 3.500 US-Dollar schwanken. ${ }^{161}$ Je nach Wunsch der Gäste konnte das Leben der Oberschicht geführt oder in die Rolle der Dienstboten geschlüpft werden. Es war zwar beliebig oft möglich, zwischen den zwei Welten zu wechseln, der Preis blieb aber derselbe - unabhängig davon, ob sich die Besucher*innen bedienen ließen oder selbst arbeiteten. ${ }^{162}$ Die Gäste lernten so gegen Bezahlung, wie in einem country house ein Bett gemacht, gekocht, serviert und geputzt wird. ${ }^{163}$

Auch wenn gerne damit geworben wurde, handelte es sich keineswegs um ein Abbild des damaligen Lebens in einem britischen stately home, sondern lediglich um eine stilisierte Inszenierung der Vergangenheit. Andrew Higson spricht im Zusammenhang mit dem Wunsch, die Vergangenheit erleben zu wollen, von nostalgia - gleichbedeutend

156 Cox, The "Downton Boom", S. 115

157 Sarah Edwards/Juliette Wilson, Do we do the past differently now? An interview with David Lowenthal, in: Consumption Markets \& Culture 17 (2014), Heft 2, S. 105-119, hier S. 108.

158 Suzannah Lipscomb, Historical Authenticity and Interpretative Strategy at Hampton Country Palace, in: The Public Historian 32 (2010), Heft 3, S. 98-119, hier S. 111.

159 Lücke/Zündorf, Einführung in die Public History, S. 82-83.

160 Korte/Paletschek, Geschichte in populären Medien, S. 15.

161 Joi-Marie McKenzie, Have the Ultimate,Downton Abbey' Experience at Dundas Castle in Scotland, in: ABC News, 30.1.2016, https://abcnews.go.com/Lifestyle/ultimate-downton-abbey-experience-dundas-castle-scotland/ story?id=36616173, eingesehen 1.5.2020; Peter Nonnenmacher, Klassenwechsel im Schloss, in: Tagesanzeiger, 15.3.2016, https://www.tagesanzeiger.ch/panorama/vermischtes/schloss-mit-klassenwechsel/story/18125072, eingesehen 1.5.2020.

162 Ebd.

163 Marc Horne, £2,500 for day as a Downton servant, in: The Times, 9.3.2016, https://www.thetimes.co.uk/article/ 2-500-for-day-as-a-downton-servant-hpvofqzwk, eingesehen 1.5.2020. 
mit einer Verklärung der Vergangenheit. ${ }^{164}$ Dementsprechend sollte diese Art der Geschichtsdarstellung und -vermittlung von Historiker*innen kritisch hinterfragt werden.

\section{Fazit}

Die vorliegende Arbeit hat sich mit den Fragen auseinandergesetzt, ob Szenische Historienserien für die Geschichtswissenschaft von Nutzen sein können und welche Auswirkungen die Fernsehserie „Downton Abbey" auf die Public History und den Filmtourismus hat. Die eingehende Beschäftigung mit dem Thema hat gezeigt, dass die eingangs aufgestellte These zum Teil verifiziert werden kann. So verdeutlicht das Beispiel "Downton Abbey", dass Szenische Historienserien ein mögliches Medium sind, um in der Öffentlichkeit Interesse für Geschichte zu wecken. Auch wenn das Verhältnis der Geschichtswissenschaft zum Film nicht unumstritten ist, handelt es sich um ein wichtiges Medium zur populären Vergangenheitsvermittlung. Vor allem das British Costume Drama, das sich auf vielfältige Weise mit der britischen Geschichte, ihrem Erbe und ihren Traditionen auseinandersetzt, prägt das kollektive Gedächtnis entscheidend und hat, wie „Downton Abbey“ demonstriert, vielfältige Auswirkungen.

Die Serie weckt bei den Zuschauer*innen den Wunsch, die Drehorte zu besuchen und hat ein gesteigertes öffentliches Interesse an den historischen Orten zur Folge. Diese Entwicklung lässt sich besonders an Highclere Castle erkennen: Während die Erhaltungskosten des Schlosses vor dem Dreh kaum gedeckt werden konnten, ist es jetzt ein beliebtes Ausflugsziel, das schrittweise renoviert wurde. Der Ort Bampton verzeichnete ebenso einen touristischen Aufschwung und versucht nun mit einem an „Downton Abbey" angelehnten Projekt die alte Grammar School zu restaurieren. Generell lässt sich ein zunehmendes Interesse an den stately homes feststellen, welche mit entsprechenden „Downton Abbey“ Ausstellungen und Erlebnispaketen darauf reagieren. Szenische Historienserien, wie „Downton Abbey", verändern also die Art und Weise, wie Vergangenheit konsumiert wird und beschleunigen bereits bestehende Trends in der musealen Präsentation. Es zeigt sich, dass eine Fernsehserie durchaus Einfluss auf die öffentliche Geschichtsdarstellung und den Tourismus haben kann. Somit kann einerseits argumentiert werden, dass Szenische Historienserien für die Geschichtswissenschaft von Nutzen sein können, da es durch das entstandene Interesse möglich wird, einem größeren Publikum Geschichte zu vermitteln und gegebenenfalls alte Gebäude zu erhalten. Andererseits muss berücksichtigt werden, dass es sich um eine verklärte Inszenierung der Vergangenheit handelt und die Grenzen zwischen seriöser Public History und Kommerz verschwimmen - meist zum Nachteil für die historischen Inhalte. 


\section{Literatur und Internetquellen}

Adams, Stephen, National Trust is 'Disney-fying' its country houses, say critics, in: The Telegraph, 29.5.2010, http://www.telegraph.co.uk/culture/culturenews/7778870/ NationalTrust-is-Disney-fying-its-country-houses-say-critics.html, eingesehen 18.4.2020.

Assmann, Aleida/Assmann, Jan, Das Gestern im Heute. Medien und soziales Gedächtnis, in: Klaus Merten/Siegfried Schmidt/Siegfried Weischenberger (Hrsg.), Die Wirklichkeit der Medien. Eine Einführung in die Kommunikationswissenschaft, Opladen 1994, S. 114-140.

Assmann, Jan, Das kulturelle Gedächtnis. Schrift, Erinnerung und politische Identität in frühen Hochkulturen, München $2018^{8}$.

Bagnoli, Lorenzo, "Downton Abbey" and the TV-induced Tourism, in: AlmaTourism. Journal of Tourism, Culture and Territorial Development 4 (2015), S. 102-116.

Bampton Beam (Hrsg.), Downton Abbey, o. D., http://www.bamptonbeam.co.uk/ downton-abbey, eingesehen 10.4.2020.

Bampton Community Archive (Hrsg.), About us, O. D., https://www.bamptonarchive. org/the-bampton-community-archive/, eingesehen 10.4.2020

Ders. (Hrsg.), Downton Abbey, O. D., https://atom.bamptonarchive.org/index.php/ downton-abbey, eingesehen 10.4.2020.

Ders. (Hrsg.), The ,Downton' Mile, o. D., http://www.downtonmile.co.uk/, eingesehen 10.4.2020.

Beeton, Sue, Film-Induced Tourism (Aspects of Tourism 76), Clevedon $2016^{2}$.

Biltmore Estate (Hrsg.), Downton Abbey: The Exhibition by the Numbers, 12.11.2019, https://www.biltmore.com/blog/downton-abbey-the-exhibition-by-the-numbers/, eingesehen 15.4.2020.

Ders. (Hrsg.), Estate History, o. D., https://www.biltmore.com/our-story/estate-history/, eingesehen 15.4.2020.

Ders. (Hrsg.), COVID-19. Health and Safety Changes, o. D., https://www.biltmore.com/ coronavirus-covid-19-update/, eingesehen 15.4.2020.

Ders. (Hrsg.), Downton Abbey: The Exhibition now open at Biltmore, 8.11.2019, https:// www.biltmore.com/news-release/downton-abbey-the-exhibition-now-open-atbiltmore/, eingesehen 15.4.2020.

Ders. (Hrsg.), Comparing Biltmore House to Downton Abbey, 18.8.2019, https://www. biltmore.com/blog/comparing-biltmore-house-to-downton-abbey-2/, eingesehen 15.4.2020.

Ders. (Hrsg.), Top 5 Downton Abbey-Related Activities at Biltmore, 7.11.2019, https:// www.biltmore.com/blog/top-5-downton-abbey-related-activities-at-biltmore/, eingesehen 15.4.2020. 
Brown, Lucy, Homosexual Lives. Representation and Reinterpretation in Upstairs, Downstairs and Downton Abbey, in: James Legott/Julie Anne Taddeo (Hrsg.), Upstairs and Downstairs. British Costume Drama Television from The Forsyte Saga to Downton Abbey, London 2015, S. 263-273.

Byrne, Katherine, New Developments in Heritage. The Recent Dark Site of Downton "Downer" Abbey, in: James Legott/Julie Anne Taddeo (Hrsg.), Upstairs and Downstairs. British Costume Drama Television from The Forsyte Saga to Downton Abbey, London 2015, S. 177-189.

Carnival Films (Hrsg.), Downton Abbey, o. D., http://www.carnivalfilms.co.uk/ downton-abbey/, eingesehen 19.3.2020.

Cox, Oliver, The "Downton Boom". Country Houses, Popular Culture, and Curatorial Culture, in: The Public Historian 37 (2015), Heft 2, S. 112-119.

David, Andrea, Downton Abbey, in: Filmtourismus. Die Welt der Drehorte, Blogbeitrag o. D., https://www.filmtourismus.de/downton-abbey/, eingesehen 10.4.2020.

Downton Abbey: The Exhibition (Hrsg.), Experience the history, the fashion and the house, o. D., https://www.downtonexhibition.com/, eingesehen 15.4.2020.

Edwards, Sarah/Juliette Wilson, Do we do the past differently now? An interview with David Lowenthal, in: Consumption Markets \& Culture 17 (2014), Nr. 2, S. 105-119.

Engler, Michael, Downton Abbey, DVD, 2960 min., London 2010-2015.

Fischer, Thomas/Schuhbauer, Thomas, Geschichte in Film und Fernsehen. TheoriePraxis-Berufsfelder (Public history-Geschichte in der Praxis), Tübingen 2016.

Gold, Tanya, Downton Abbey. class and distinction, in: The Guardian, 13.9.2011, https://www.theguardian.com/tv-and-radio/2011/sep/13/downton-abbey-class-anddistinction, eingesehen 22.3.2020.

Groot, Jerome de, Foreword, in: James Legott/Julie Anne Taddeo (Hrsg.), Upstairs and Downstairs. British Costume Drama Television from The Forsyte Saga to Downton Abbey, London 2015, S. IX-XII.

Hardiman, Robert, Defending the real Downton Abbeys. Why Britain's stately homes are struggling, in: The Spectator, 9.3.2013, https://www.spectator.co.uk/article/ defending-the-real-downton-abbeys, eingesehen 5.4.2020.

Hasenberg, Peter, Eine einfachere Welt? Familienbilder und gesellschaftlicher Wandel in der britischen TV-Serie Downton Abbbey, in: Peter Hasenberg/Markus Leniger/Reinhold Zwick (Hrsg.), Familienbilder. Reflexionen und Konstruktionen zum Thema Familie im aktuellen Spielfilm (Religion, Film und Medien 4), Marburg 2018, S. 173-209.

Heath, Stephen, Representing Television, in: Patricia Mellankamp (Hrsg.), Logics of Television. Essays in Cultural Criticism, Bloomington 1990.

Hellemans, Jorden, Film Induced Tourism. Television Series, Diss., Universidad de Murcia 2014 
Highclere Castle (Hrsg.), Our Ticket System, o. D., https://www.highclerecastle.co.uk/ our-ticket-system-pre-book-and-walk, eingesehen 4.4.2020.

Ders. (Hrsg.), Castle Tour, Egyptian Exhibition \& Afternoon Tea, o. D., https://highclerecastleshop.co.uk/categories/castle-tour-exhibition-gardens-and-afternoon-tea, eingesehen 4.4.2020.

Ders. (Hrsg.), Highclere Castle History Festival Weekend, O. D., https://highclerecastleshop.co.uk/highclere-castle-history-festival, eingesehen 4.4.2020.

Ders. (Hrsg.), Summer 2020 Admission Tickets, o. D., https://highclerecastleshop.co.uk/ categories/summer-2020-admission-tickets, eingesehen 4.4.2020.

Ders. (Hrsg.), Living in a Castle, o. D., https://highclerecastleshop.co.uk/categories/livingin-a-castle, eingesehen 4.4.2020.

Ders. (Hrsg.), London Lodge, o. D., https://www.highclerecastle.co.uk/london-lodge, eingesehen 4.4.2020.

Ders. (Hrsg.), Grotto Lodge, o. D., https://www.highclerecastle.co.uk/grotto-lodge, eingesehen 4.4.2020.

Ders. (Hrsg.), Home, o. D., https://www.highclerecastle.co.uk/, eingesehen 4.4.2020.

Ders. (Hrsg.), YouTube, O. D., https://www.youtube.com/channel/UCLbmDFj2sQV4n4R1QaSfZlg/featured, eingesehen 5.4.2020.

Ders. (Hrsg.), Facebook, o. D., https://www.facebook.com/HighclereCastleOfficial/, eingesehen 5.4.2020.

Ders. (Hrsg.), Twitter, o. D., https://twitter.com/HighclereCastle, https://www.instagram. com/highclere_castle/, eingesehen 5.4.2020.

Ders. (Hrsg.), Instagram, o. D., https://www.instagram.com/highclere_castle/?hl=de, eingesehen 5.4.2020.

Ders. (Hrsg.), Highclere Castle launches New App on iTunes and GoglePlay, 5.1.2016, https://www.highclerecastle.co.uk/news/highclere-castle-launches-new-app-itunesand-google-play, eingesehen 5.4.2020.

Higson, Andrew, English heritage, English cinema. Costume drama since 1980, Oxford 2003.

Ders., Film England. Culturally English filmmaking since the 1990s, London-New York 2011.

Ders., Nostalgia is not what it used to be. Heritage films, nostalgia websites and contemporary consumers, in: Consumption Markets \& Culture 17 (2014), Heft 2, S. 120-142.

HM Treasury (Hrsg.), Reform of film taxes incentives. Promoting the sustainable production of culturally British films, Juli 2005, www.hm-treasury.gov.uk/d/filmcondocv1.pdf, eingesehen 18.3.2009. 
Horne, Marc, $£ 2,500$ for day as a Downton servant, in: The Times, 9.3.2016, https://www. thetimes.co.uk/article/2-500-for-day-as-a-downton-servant-hpvOfqzwk, eingesehen 1.5.2020.

Hughes, Tammy, Downton Abbey is open for business. Lord and Lady Carnarvon re-open Highclere Castle to the public, in: The Daily Mail, 21.1.2017, https://www.dailymail.co.uk/ news/article-4142220/Downton-Abbey-set-open-public.html, eingesehen 5.4.2020.

Ihring, Silvia, Ein Zimmer in „Downton Abbey“ kann man jetzt auf Airbnb mieten, in: Welt, 1.10.2019, https://www.welt.de/icon/unterwegs/article201175490/Airbnb-bietetUebernachtung-im-Schloss-aus-Downton-Abbey.html, eingesehen 4.4.2020.

Jacobs, Laura, Upstairs Timelessness, Downstairs Precision, in: The Wall Street Journal, 8.7.2014, http://online.wsj.com/articles/exhibition-review-upstairstimelessnessdownstairs-precision-1404861153, eingesehen 13.4.2020.

Lady Carnarvon (Hrsg.), The Downton Abbey Concert, 10.12.2018, https://www.ladycarnarvon.com/the-downton-abbey-concert/, eingesehen 4.4.2020.

Dies. (Hrsg.), The official Website of the Countess of Carnarvon, o. D, https://www. ladycarnarvon.com/, eingesehen 4.4.2020.

Dies. (Hrsg.), About, o. D, https://www.ladycarnarvon.com/about/, eingesehen 4.4.2020.

Dies. (Hrsg.), Blog, o. D, https://www.ladycarnarvon.com/blog/, eingesehen 5.4.2020.

Lipscomb, Suzannah, Historical Authenticity and Interpretative Strategy at Hampton Country Palace, in: The Public Historian 32 (2010), Heft 3, S. 98-119.

Lowenthal, David, Why the Past Matters, in: Heritage \& Society 4 (2011), Heft 2, S. 159-171.

Legott, James/Taddeo, Julie Anne, Indroduction, in: James Legott/Julie Anne Taddeo (Hrsg.), Upstairs and Downstairs. British Costume Drama Television from The Forsyte Saga to Downton Abbey, London 2015, S. XV-XXX.

Mattisson, Jane, Downton Abbey. A Cultural Phenomenon. History for the Many, in: SIC. Journal of Literature, Culture and Literary Translation 5 (2014), S. 1-27, https://www. sic-journal.org/Article/Index/291, eingesehen 1.5.2020.

McKenzie, Joi-Marie, Have the Ultimate 'Downton Abbey' Experience at Dundas Castle in Scotland, in: ABC News, 30.1.2016, https://abcnews.go.com/Lifestyle/ultimate-downtonabbey-experience-dundas-castle-scotland/story?id=36616173, eingesehen 1.5.2020.

Micklefield, Viv, Lady Carnarvon on Downton Abbey, life at Highclere Castle and her blog, in: Hampshire Life, 15.1.2018, https://www.hampshire-life.co.uk/people/ladycarnarvon-on-downton-abbey-life-at-highclere-castle-and-her-blog-1-5355878, eingesehen 4.4.2020.

Monk, Claire, The British heritage-film debate revisited, in: Claire Monk/Amy Sargeant (Hrsg.), British historical cinema. The history, heritage and costume film (British popular cinema), London-New York 2002, S. 176-198. 
Murphy, Robert (Hrsg.), The British Cinema Book, London $2018^{3}$.

Nonnenmacher, Peter, Klassenwechsel im Schloss, in: Tagesanzeiger, 15.3.2016, https://www.tagesanzeiger.ch/panorama/vermischtes/schloss-mit-klassenwechsel/ story/18125072, eingesehen 1.5.2020.

Oxfordshire Cotswolds Tourism (Hrsg.), Downton Abbey film locations, o. D., https:// www.oxfordshirecotswolds.org/plan-your-visit/towns-and-villages/downton-abbeyfilm-locations, eingesehen 10.4.2020.

Korte, Barbara/Paletschek, Sylvia, Geschichte in populären Medien und Genres. Vom historischen Roman zum Computerspiel, in: Barbara Korte/Sylvia Paletschek (Hrsg.), History Goes Pop. Zur Repräsentation von Geschichte in populären Medien und Genres (Historische Lebenswelten in populären Wissenskulturen/History in Popular Cultures 1), Bielefeld 2009, S. 9-60.

Paul, Gerhard, Von der historischen Bildkunde zur Visual History. Eine Einführung, in: Gerhard Paul (Hrsg.), Visual History. Ein Studienbuch, Göttingen 2006, S. 7-36.

Schama, Simon, Why Americans have fallen for Snobby 'Downton Abbey', in: Newsweek, 16.1.2012, https://www.newsweek.com/why-americans-have-fallen-snobby-downtonabbey-64157, eingesehen 22.3.2020.

Sommer, Andreas, Geschichtsbilder und Spielfilme. Eine qualitative Studie zur Kohärenz zwischen Geschichtsbild und historischem Spielfilm bei Geschichtsstudierenden (Geschichtskultur und historisches Lernen 5), Berlin 2010.

Sperati, J. P./Schreiner, Sabine, Downton Abbey on Location. An unofficial Review \& Guide to the Locations used, Sawston-Cambridge 2017.

Steinecke, Albrecht, Filmtourismus, Konstanz-München 2016.

Thomas, Gina, Eine Nation flüchtet in die Vergangenheit, in: Frankfurter Allgemeine, 22.9.2013, https://www.faz.net/aktuell/feuilleton/medien/fernsehserie-downton-abbeyiv-eine-nation-fluechtet-in-die-vergangenheit-12583246.html, eingesehen 22.3.2020.

VisitBritain (Hrsg.), Global partnership campaigns, O. D., https://www.visitbritain.org/ global-partnership-campaigns, eingesehen 24.3.2020.

Ders. (Hrsg.), Bampton, o. D., https://www.visitbritain.com/de/de/bampton, eingesehen 10.4.2020.

Welzer, Harald, Das soziale Gedächtnis, in: Harald Welzer (Hrsg.), Das soziale Gedächtnis. Geschichte, Erinnerung, Tradierung, Hamburg 2001, S. 11-14.

West, Nancy, The Worlds of Downton Abbey, in: South Atlantic Review 80 (2015), Heft 3, S. $215-233$.

Winterthur Museum (Hrsg.), Costumes of Downton Abbey, o. D., http://www.winterthur. org/exhibitions-events/exhibitions/past-exhibitions/costumes-of-downton-abbey/, eingesehen 13.4.2020. 
Ders. (Hrsg.), Costumes of Downton Abbey, O. D., http://www.winterthur.org/ exhibitions-events/exhibitions/past-exhibitions/costumes-of-downton-abbey/, eingesehen 13.4.2020.

Whittaker, Alexandra, Downton Abbey Exhibit coming to Chicago, in: Medill Reports Chicago, 27.1.2016, https://news.medill.northwestern.edu/chicago/downton-abbeyexhibit-coming-to-chicago/, eingesehen 13.4.2020.

Zaschke, Christian, Reich und schön, in: Süddeutsche Zeitung, 15.12.2017, https:// www.sueddeutsche.de/medien/das-empire-gastiert-in-new-york-reich-und-schoen1.3793889? reduced=true, eingesehen 22.3.2020.

Verena Hechenblaikner ist Masterstudentin der Geschichtswissenschaften an der Universität Innsbruck und studentische Mitarbeiterin am Institut für Zeitgeschichte. verena.hechenblaikner@uibk.ac.at

\section{Zitation dieses Beitrages}

Verena Hechenblaikner, Downton-Mania. Die Fernsehserie „Downton Abbey“ und ihre Auswirkung auf die Public History und den Filmtourismus, in: historia.scribere 13 (2021), S. 31-56, [http://historia.scribere.at], eingesehen 22.6.2021 (=aktuelles Datum).

Creative Commons Licences 3.0 Österreich unter Wahrung der Urheberrechte der Autorlnnen. 